\title{
A!
}

This is an electronic reprint of the original article.

This reprint may differ from the original in pagination and typographic detail.

Makowska, Michalina; Pellinen, Terhi

\section{Qualitative detection of the presence of Gilsonite in the bituminous blends based on thin layer chromatography}

Published in:

RILEM 252-CMB: RILEM 252-CMB Symposium on Chemo Mechanical Characterization of Bituminous Materials

DOI:

10.1007/978-3-030-00476-7_38

Published: 01/01/2019

Document Version

Peer reviewed version

Please cite the original version:

Makowska, M., \& Pellinen, T. (2019). Qualitative detection of the presence of Gilsonite in the bituminous blends based on thin layer chromatography. In L. Poulikakos, A. Cannone Falchetto, M. Wistuba, B. Hofko, L. Porot, \& H. Di Benedetto (Eds.), RILEM 252-CMB: RILEM 252-CMB Symposium on Chemo Mechanical Characterization of Bituminous Materials (pp. 242-247). (RILEM Bookseries; Vol. 20). SPRINGER. https://doi.org/10.1007/978-3030-00476-7_38

This material is protected by copyright and other intellectual property rights, and duplication or sale of all or part of any of the repository collections is not permitted, except that material may be duplicated by you for your research use or educational purposes in electronic or print form. You must obtain permission for any other use. Electronic or print copies may not be offered, whether for sale or otherwise to anyone who is not an authorised user. 


\title{
Qualitative detection of the presence of Gilsonite in the bituminous blends based on thin layer chromatography
}

\author{
Michalina Makowska, Terhi Pellinen
}

Department of Civil Engineering, Aalto University, Espoo, Finland, michalina.makowska@aalto.fi

\begin{abstract}
The natural asphalts, of which Gilsonite is a representative, are modifiers used to increase the stiffness modulus of the asphalt concrete. For quality control purpose, recognizing if such material is present in the final blend is of an interest. The thin layer chromatography using a flame photometric detector (FPD), in addition to the typical flame ionization detector, was demonstrated hereby as a viable analytical tool for this problem. Gilsonite also contains the material soluble in solvent used in the development of the fraction referred to as aromatics. However, for straight run bitumen and Gilsonite the color of that fraction is different as well as their mobility. Due to the lower mobility of Gilsonite molecules on the stationary phase, the elution stops at different position than for fresh bitumen, convoluting the signal in the chromatogram region typically assigned for resins. The presence of Gilsonite is identifiable visually after the second development bath, but also with FPD from the final chromatogram.
\end{abstract}

Keywords Thin Layer Chromatography, Flame Photometric Detector, Gilsonite

\section{Introduction}

There are specific applications in paving industry where the high stiffness modulus of asphalt is preferred such as the bound base courses of the heavily loaded roads or the highly rut resistant surface layers. The stiffness modulus of asphalt concrete relates to the stiffness modulus of bitumen (Pellinen et al. 2007). In order to improve resistance to the permanent deformation of asphalt concrete and to increase its modulus, different bitumen modifiers have been investigated over the 
years, e.g. natural asphalts (NA) (Widyatmoko and Elliott 2008), recycled asphalt shingles (RAS), and recycled asphalt pavements (RAP). However, when certain modifiers such as NA mix with the bitumen, the detection of the modifier from the bitumen blend becomes challenging. Considering three blends with such modifiers at an equal stiffness of the binder, polymer modification (e.g. through RAS) is identifiable and quantifiable using infrared spectroscopy (Zofka et al. 2015), while sadly distinguishing between NA and aged bitumen poses challenge.

However, there is a chemical difference between the bitumen and NA, as both the straight run bitumen and the NA differ in polarity as witnessed by their Hansen Solubility Parameters (HSP) (Redelius 2000; Hansen 2007), at least as reported for Gilsonite and various bitumen types. The HSP determination methodology does not allow for quantification of the amount of material, which becomes miscible in a chosen solvent, thus having lower applicability for blend analysis. Potentially, Thin Layer Chromatography (TLC) could allow differentiation between the two materials and even their blends, as it incorporates both information about solubility and polar interaction with the stationary phase (Spangenberg et al. 2011), while the signal can be quantified by the use of detectors. Some of the most applied detectors are based on the light absorbance because the chromatography deals with separation of the mixtures of the colorful compounds (chroma - $g r$. color). Since the separated compounds are often organic, there are also the flame ionization detectors (FID) coupled with the technique, which scintillate the carbon cations using the change in conductivity of the exhaust gas.

The equipment typically used in the bituminous materials industry to perform the TLC is IATROSCAN (Ogasawa et al. 2002). It can be equipped in the flame ionization detector (FID) or with Flame Photometric Detector (FPD). The FPD is in principle an FID, extended by a filter and a photomultiplier tube (Ogasawa et al. 2002). Due to the increasing interest in sulfur (S) distribution in bituminous fractions and their effect on bitumen properties (Makowska et al. 2017; McKenna et al. 2013) the FPD with a filter at $394 \mathrm{~nm}$ wavelength is explored (S-FPD).

The bitumen divides into fractions based on the polarity of the molecules with various techniques, and the Corbett technique is the most standard division into Saturates, Aromatics, Resins and Asphaltenes (SARA) (Lehto 1988). Currently, the TLC-FID based SARA fractionation technique is dominant due to its simplicity and low solvent requirement. However, the fractions of the column chromatography (two types of stationary phase) are not exactly the same as those obtained in TLC-FID (one stationary phase) and it was proposed that the products of those separations be named differently (Masson et al. 2001). Additionally, Jiang et al. (2008) pointed out that the fraction obtained in column chromatography, when eluted in TLC-FID program can contribute to more than one peak.

Moreover, comparing the SARA fraction results from TLC-FID performed in different laboratories is challenging due to the use of the differing solvents and times of elution (often not reported in literature) (Masson et al. 2001; Higuerey et al. 2002; Tabatabaee and Kurth 2017; Paliukaite et al. 2014). The laboratories choose the method for the inside quality control of the product, or for the compari- 
son between the products in development stage, based on inside correlations (Lehto 1988; Teugels and Zwijsen 1991). The elution regime is stable within a laboratory to assure the good repeatability, but the economic factors seem to play role in the choice of the parameters in the method (e.g. cost of the rods, length of the process).

Nevertheless, the technique is still providing information about the solubility and the polarity, which is quantifiable. As an example, hereby article was inspired by an observation of a different elution pattern from samples of bitumen modified with Gilsonite (GIL) in the consecutive development steps of typical SARA fractioning through TLC technique. In the development, in so-called second bath, by n-heptan/toluene $(80: 20 \%$ vol.), which for straight run bitumen elutes to the height of about $5 \mathrm{~cm}$ from origin, the GIL sample was staining the rods only close to the original spot (approximately to the height of 2-3 cm). It also had a different color (yellow for 70/100 and brown-red for GIL). This observation could be helpful during forensic analysis of the asphalt concrete samples in terms of identification of the presence of GIL as a modifier. Thus, this article investigates how the incorporation of the S-FPD supports qualitative identification of Gilsonite as a modifier in the bituminous blends.

\section{Materials and Methodology}

The natural asphalt, namely Gilsonite (GIL), and paving grade bitumen 70/100 were used in the analysis. Gilsonite is allowed in the Finnish Asphalt Specifications (Finnish Pavement Technology Advisory Council 2017) as a modifier of asphalt concrete to improve resistance to permanent deformation. It is added at about $0.5 \%$ wt. per mass, which makes the approximate ratio between bitumen and Gilsonite in the asphalt concrete about 90:10 by weight and such blend was prepared as a reference for investigation (BLEND).

A solution of approximately $1 \%$ vol. of material in chloroform was prepared. $2 \mu \mathrm{l}$ drop was spotted on each rod. The material studied was then spotted on the rods and after that dried in nitrogen flow for 10 minutes. One rod in the series stays clean and acts as a reference to evaluate if the full solvent evaporation occurred (Figure 1).

A series of 3 baths was prepared: 1) n-heptan -30 minutes $(9 \mathrm{~cm}), 2) \mathrm{n}$ heptan/toluene (80:20\% vol.) - 10 minutes $(5 \mathrm{~cm}), 3)$ dichloromethane/methanol $(95: 5 \%$ vol. $)-3$ minutes $(2.5 \mathrm{~cm})$. The investigated materials were measured in three series (Figure 2). The first was analyzed according to the classical TLC-FID methodology where bath 1,2 and 3 were used (3B); the second group was developed only in bath 1 and 2 (2B) and the third group was developed in bath number 1 only (1B) (inspired by the works of Lu et al. (2008). Three repetitions were performed for each sample and each series. Unfortunately, one of the pure Gilsonite repetitions in 3B failed (Figure 1). Between immersions in each bath and after- 
wards, rods were removed from development tanks and dried in pure nitrogen flow for a minimum of 5 minutes. After the development the full pyrolysis scan (30 seconds/rod) was applied on the rods for all of the groups, using Iatroskan MK-6/6S, equipped in the typical Flame Ionisation Detector, as well as Flame Photometric Detector focused on the detection of the sulfurous compounds.

a)
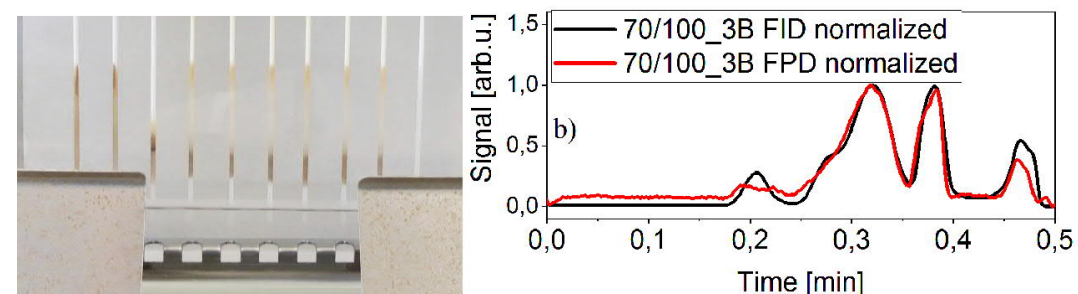

Fig.1. a) The picture taken during the development in the third solvent (from the left): GIL (rods no. 1-3), bitumen 70/100 (rods no. 4-6), bitumen BLEND (rods no. 7-9), reference (rod no. 10); b) an example of the signals obtained from FID and FPD detectors simultaneously from one rod after normalization

\section{Results and discussion}

As presented in Figure 2c the final chromatogram is dividable into four major peaks representing the saturates, aromatics, resins and asphaltenes, or areas where such peaks should be observed. However, due to the overlap of the GIL signal from second bath (Figure 2b), the position of the valley between the resin and asphaltene characteristic peak is altered for GIL and its blends. The phenomenon demonstrated in Figure 2 suggests that the molecules soluble in the same solvent of second bath from GIL are expressing lower mobility on the stationary phase (higher polar interaction with solid phase or different size).

As can be seen the majority of the sulfur signal is coming from the peak formed as a result of 2B (Figure 1) (Ogasawa et al. 2002). Incidentally, it is the peak imposed in the mentioned valley region. The blend of the GIL and the fresh bitumen is indeed giving a slight peak by the S-FPD in that region (Figure 3), which could prove useful for the qualitative recognition of GIL presence.

\section{Conclusion}

The thin layer chromatography separation of bitumen is questionable in terms of comparison to the SARA fractionation obtained through the column chromatography technique. 


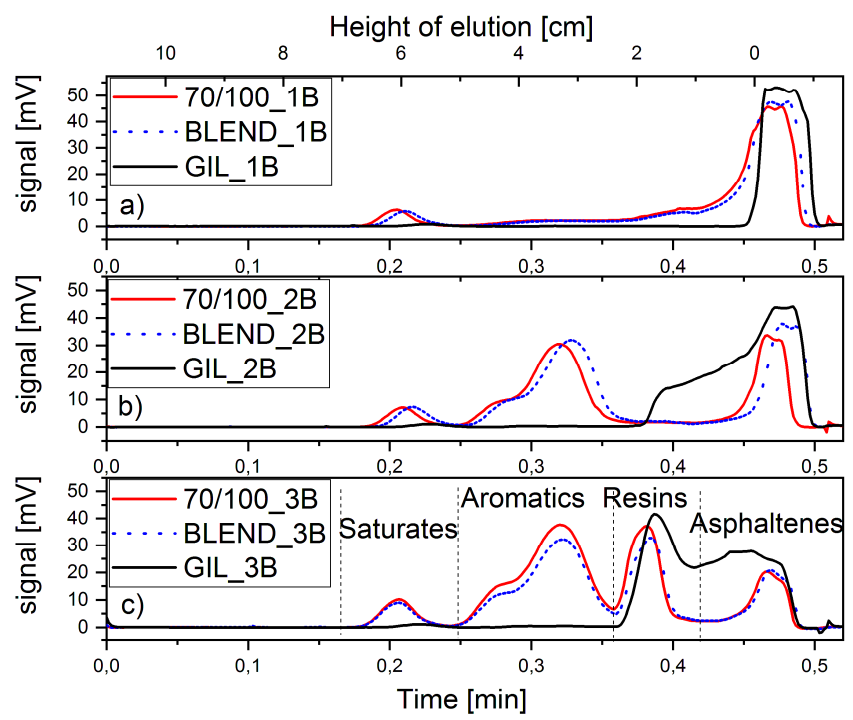

Fig. 2. The chromatogram from TLC-FID of 70/100, GIL and BLEND after a) $1 B$, b) $2 B$ and c) $3 \mathrm{~B}$ with visual division of SARA fractions typically assigned in the procedure

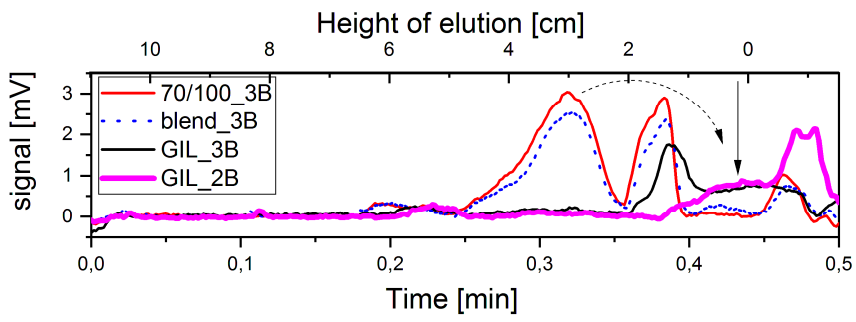

Fig. 3. The chromatogram from TLC-FPD (Sulphur) of 70/100_3B, Gilsonite (GIL_3B and GIL_2B) and blend of the two in the 90:10 ratio after elution in three baths (BLEND_3B), where the arrows indicate region of interest

However, it is still a useful technique to distinguish between the bitumen samples, as demonstrated on the example of analysis of the blends of the straight run bitumen and Gilsonite, employing both the Flame Ionization Detector and the Flame Photometric Detector (394 nm).

The molecules developed by the solvent combination assigned to the signal of aromatics in the straight run bitumen, solubilized the molecules of Gilsonite. However, their elution upwards the rod was hindered due to their interaction with stationary phase of TLC rods, imposing their signal in the region typically assigned to the resin signal. This fact is observable by an operator due to the difference in color, but also as demonstrated by the utilization of S-FPD detector. The research demonstrated how this analytical technique is applicable for the qualitative recognition of the Gilsonite presence in the bituminous blends. 


\section{References}

Finnish Pavement Technology Advisory Council (2017) Finnish Asphalt Specifications 2017. Helsinki: Premedia Helsinki Oy.

Hansen C (2007) Hansen Solubility Parameters - A user's Handbook. Boca Raton, Fl: CRC Press.

Higuerey I, Orea M \& Pereira P (2002) Estimation Of Visbroken And Selective Catalytic Steam Cracked Product Stability Using Iatroscan TLC-FID. Fuel Chemistry Division Preprints, 47(2):656-658.

Jiang C, Larter S, Noke K \& Snowdown L (2008) TLC-FID (Iatroscan) analysis of heavy oil and tar sand samples. Organic Geochemistry 39:1210-1214.

Lehto E (1988) Bitumin fraktiointi TLC/FID-menetelmällä [translation: Bitumen fractionation by TLC-FID method], University of Oulu, Oulu.

Lu X, Kalman B \& Redelius P (2008) A new test method for determination of wax content in crude oils, residues and bitumens. Fuel, 87:1543-1551.

Makowska M, Hartikainen A \& Pellinen T (2017) The oxidation of bitumen witnessed in-situ by infrared spectroscopy. Materials and Structures, 50:189.

Masson J-F, Price T \& Collins P (2001) Dynamics of Bitumen Fractions by Thin-Layer Chromatography/Flame Ionization Detection. Energy \& Fuels, 15:955-960.

McKenna AM, Marshall AG \& Rodgers RP (2013) Heavy Petroleum Composition. 4. Asphaltene Compositional Space. Energy Fuels 27:1257-1267.

Ogasawa M, Tsuruta K \& Arao S (2002) Flame photometric detector for thin-layer chromatography. Journal of Chromatography A, 973(1-2):151-158.

Paliukaite M, Vaitkus A \& Zofka A (2014) Evaluation of bitumen fractional composition depending on the crude oil type and production technology. Vilnus, Lithuania22-23 May 2014

Pellinen T, Zofka A, Marasteanu M \& Funk N (2007) Asphalt Mixture Stiffness Predictive Models (With Discussion). Journal of the Association of Asphalt Paving Technologists: From the Proceedings of the Technical Sessions 76:575-626.

Redelius P (2000) Solubility parameters and bitumen. Fuel 79:27-35.

Spangenberg B, Poole C \& Weins C (2011) Quantitative Thin-Layer Chromatography (A practical Survey). Berlin Heidelberg: Springer-Verlag .

Tabatabaee H \& Kurth T (2017) Analytical investigation of the impact of a novel bio-based recycling agent on the colloidal stability of aged bitumen. Road Materials and Pavement Design, Volume 18, Issue sup2: EATA 2017, p 131-140.

Teugels W \& Zwijsen M (1991) Analysis of the generic composition. Appraisal of the Iatroskan method. Paper presented at the International symposium chemistry of bitumens, Rome, 1991

Widyatmoko I \& Elliott R (2008) Characteristics of elastomeric and plastomeric binders in contact with natural asphalts. Construction and Building Materials, 22:239-249.

Zofka A, Maliszewska D, Maliszewski M \& Boratynski J (2015) Application of FTIR ATR method to examine the polymer content in the modified bitumen and to assess susceptibility of bitumen to ageing. Road and Bridges, p 163-174. 